\title{
THE USE OF POLYTHENE BAGS FOR DELIVERY-ROOM THERMOREGULATION IN PREMATURE BABIES ACROSS ENGLAND
}

\author{
A. Godhamgaonkar, V. Parmar, E. Kyte, M. Sarwar \\ Neonatology, St. Mary's Hospital, Manchester, UK, Manchester, UK
}

\begin{abstract}
Introduction: Polythene bags have been showed to be effective in temperature control of premature babies in the delivery room. Both, the Advanced Paediatric Life Support and the Newborn Life Support courses recommend its use. Nonetheless, variation in practices still exists. In addition, the MHRA has previously advised to avoid using non CE-marked products for clinical purposes.
\end{abstract}

Aims: To establish if a formal thermoregulation policy for premature babies exists in neonatal units across England. To determine the usage of different types of polythene bags and the gestational age limits. To ascertain the use of transwarmer as an adjunct for thermoregulation.

Methods: A structured telephone survey of 131 neonatal units across England was performed. The survey consisted of questions related to the existence of a thermoregulation policy, use of polythene bags, their type, gestational age limits and use of transwarmer.

Results: There was $100 \%$ response rate. 129 neonatal units $(98.5 \%)$ had a formal thermoregulation policy. 124 units $(94.65 \%)$ used plastic bags of which 86 units (66\%) used food grade and 38 units (29\%) used purpose made bags. 7 units did not use plastic bags of which 4 used transwarmer alone, 2 used bubble wrap and 1 unit did not use either. 29 neonatal units used both plastic bags and transwarmer for preterm deliveries.

Conclusions: Polythene bags are extensively used by neonatal units across England. Nonetheless, most neonatal units are using non- $C E$ marked polythene bags despite advice from the MHRA. 